\title{
Dynamics and regulation of lipid droplet formation in lipopolysaccharide (LPS)-stimulated microglia
}

\author{
Armen Khatchadourian ${ }^{a}$, Simon D. Bourque ${ }^{\mathrm{b}}$, Vincent R. Richard ${ }^{\mathrm{b}}$, \\ Vladimir I. Titorenko ${ }^{\mathrm{b}}$, Dusica Maysinger ${ }^{\mathrm{a}, *}$ \\ a Department of Pharmacology and Therapeutics, McGill University, Montreal, QC, Canada H3G 1Y6 \\ b Department of Biology, Concordia University, Montreal, QC, Canada H4B 1R6
}

\section{A R T I C L E I N F O}

\section{Article history:}

Received 4 August 2011

Received in revised form 4 December 2011

Accepted 10 January 2012

Available online 28 January 2012

\section{Keywords:}

Lipid droplets

Microglia activation

Lipopolysaccharides

Perilipin-2

JNK phosphorylation

p38 phosphorylation

\begin{abstract}
A B S T R A C T
Lipid droplets (LDs) are neutral lipid-rich organelles involved in many cellular processes. A well-known example is their accumulation in leukocytes upon activation by pro-inflammatory stimuli such as lipopolysaccharides (LPS) derived from gram-negative bacteria. A role of LDs and LD-associated proteins during inflammation in the brain is unknown, however. We have now studied their dynamics and regulation in microglia, the resident immune cells in the brain. We find that LPS treatment of microglia leads to the accumulation in them of LDs, and enhancement of the size of LDs. This induction of LDs was abolished by triacsin C, an inhibitor of triglyceride biosynthesis. LPS strongly activated c-Jun N-terminal kinase (JNK) and p38 MAPK stress signaling pathways and increased the expression of LD-associated protein perilipin-2 (ADRP) in a time-dependent manner. Immunostaining showed that perilipin-2 in LPS-treated microglia predominantly colocalized with LDs. Inhibitors of p38 $\alpha / \beta$ (SB203580) and PI3K/Akt pathway (LY294002), but not that of JNK (SP600125), reduced LPS-induced LD accumulation and eliminated the activating effect of LPS on perilipin-2. In addition, cytosolic phospholipase $\mathrm{A}_{2}\left(\mathrm{CPLA}_{2}-\alpha\right)$, a key enzyme for arachidonic acid release, colocalized with LPS-induced LDs. These observations suggest that LDs may play an important role in eicosanoid synthesis in activated microglia; they provide a novel insight into the regulation of LDs in inflammatory cells of the brain and point to a potential role of p38 $\alpha / \beta$ in LPS-induced LD accumulation. Collectively, our findings imply that LD formation and perilipin-2 induction could be microglial biomarkers of inflammation in the central nervous system.
\end{abstract}

(c) 2012 Elsevier B.V. All rights reserved.

\section{Introduction}

Lipid droplets (LDs), also called lipid bodies, are dynamic intracellular organelles that contain a core rich in neutral lipids, such as triglycerides (TG) and cholesteryl esters [1]. Their surface is composed of a phospholipid monolayer with a unique phospholipid and fatty acid composition [2]. Initially considered as inert neutral lipidstorage compartments, LDs have become intensively studied organelles, implicated, for example, in the pathology of atherosclerosis, obesity, insulin resistance, type 2 diabetes [3], hepatic steatosis [4], cardiovascular

Abbreviations: LD, lipid droplet; TG, triglyceride; TLR4, Toll-like receptor 4; LPS, lipopolysaccharides; ADRP, adipocyte differentiation-related protein; CNS, central nervous system; NO, nitric oxide; MAPK, mitogen-activated protein kinases; JNK, c-Jun N-terminal kinase; OA, oleic acid; BODIPY 493/503, 4,4-difluoro-1,3,5,7,8pentamethyl-4-bora-3a,4a-diaza-s-indacene; PI3K, phosphatidylinositol 3-kinase; MKK, MAPK kinase; AP-1, activator protein-1; FFA, free fatty acid; $c P L A_{2}-\alpha$, cytosolic phospholipase $\mathrm{A}_{2}$ alpha

* Corresponding author at: Department of Pharmacology and Therapeutics, McGill University, 3655 Promenade Sir William Osler, Montreal, QC, Canada H3G 1Y6. Tel.: + 1514398 1264; fax: + 15143986690 .

E-mail address: dusica.maysinger@mcgill.ca (D. Maysinger). disease, and inflammatory disorders [5,6]. The formation of LDs is a precisely regulated and inducible process which can be triggered by a variety of extracellular cues such as growth factors, long-chain fatty acids [7], oxidized low density lipoproteins [8] or by various environmental insults such as oxidative stress $[9,10]$ and inflammatory stimuli [11]. LDs are rapidly induced under inflammatory conditions in leukocytes. In experimental models of sepsis, the interaction of bacterial lipopolysaccharide (LPS) with Toll-like receptor 4 (TLR4) leads to a considerable accumulation of LDs in macrophages [12]. LDs were shown to contain esterified arachidonic acid in their phospholipid and neutral lipid pools and proposed to be intracellular sites involved in the metabolism of arachidonic acid into eicosanoids [13,14]. Cytosolic phospholipase $A_{2}-\alpha\left(C P L A_{2}-\alpha\right)$, also called group IVA PLA $A_{2}$, is a central enzyme for the release of arachidonic acid from phospholipids and has been found to co-localize with LDs [15]. $\mathrm{CPLA}_{2}-\alpha$ activation and release of proinflammatory lipid mediators are regulated by TLR4 signaling in LPSactivated macrophages [16].

The surface of LDs is coated with various proteins, many of which regulate their formation, growth or turnover [17]. Members of the PAT family proteins, namely perilipin, adipocyte differentiationrelated protein (ADRP) and tail-interacting protein $47 \mathrm{kDa}$ (TIP47), 
share extensive amino acid sequence in their amino termini (PAT domain) and bind to LDs [17]. A recently implemented new nomenclature for the PAT proteins has changed the names of perilipin, ADRP and Tip 47 to perilipin 1, 2 and 3, respectively [18]. Perilipin-2 (ADRP) and perilipin-3 (Tip 47) are ubiquitously expressed in different cell types, including macrophages $[19,20]$. Perilipin-2 is a prominent LD-associated protein, whose expression level rises concomitantly with an increase in both LD abundance and TG level [21]. Perilipin-2 is a key regulator of LD formation and has been investigated in the context of metabolic syndrome and inflammatory diseases [5].

The present study sought to advance our understanding of the physiological roles of perilipin-2 and LDs in microglia, the resident macrophage population of the central nervous system (CNS) [22]. Microglia are of myeloid origin and share certain phenotypic characteristics with macrophages in their activated form. Upon activation microglia express cell surface markers that are also present on macrophages [23]. They respond rapidly to pathological challenges in the CNS by acquiring a reactive state and secreting proinflammatory cytokines, nitric oxide (NO) and reactive oxygen species [22,24]. Importantly, TLR4-initiated signaling underlies microglia-induced inflammation in the CNS [25]. LPS-induced activation of TLR4 triggers an activating downstream signal to mitogen-activated protein kinases (MAPK), c-Jun N-terminal kinase (JNK) and p38 [25]. Phosphorylation of p38 and JNK by upstream MAPK kinases leads to activation of transcription complex activator protein -1 (AP-1), which then induces the expression of proinflammatory cytokines [26]. In LPS-treated macrophages, stimulation of the binding of AP- 1 to the Ets/AP- 1 binding site results in increased perilipin-2 expression [27]. Although the effect of LPS on perilipin-2 protein expression in macrophages has been previously examined $[27,28]$, this has not been investigated in microglia.

In the current study, we investigated the effects of LPS on LD status and perilipin-2 expression in murine N9 microglia cells. These cells express TLR4 and many other pathogen recognition receptors and rapidly respond to bacterial endotoxins [29]. The effect of LPS was compared with that of oleic acid (OA), a major plasma fatty acid [30]. In cells, OA is esterified and stored as triglyceride inside LDs. OA and other long chain fatty acids induce LD formation and perilipin-2 expression [7,31]. In the brain, $\mathrm{OA}$ is synthesized by astrocytes and acts as a neurotrophic factor for neurons [32]. Although induction of LD formation and perilipin-2 expression in response to $\mathrm{OA}$ has been assessed in different cell types, it has not been studied so far in microglia.

We hypothesized that stimulation of microglia with LPS may alter LD status and perilipin-2 expression via JNK and/or p38 MAPK. We show that LPS stimulation greatly increases microglial LD and TG content and induces perilipin-2 expression. We also demonstrate that pharmacological inhibition of p38 downregulates perilipin-2 expression and LD accumulation. OA-driven LD accumulation was not accompanied by p38 or JNK activation. In order to better understand the role of microglial LDs in the production of proinflammatory lipid mediators, we examined colocalization of $\mathrm{CPLA}_{2}-\alpha$ with LDs. Our findings imply that LPS-driven changes in LD biogenesis and perilipin-2 expression could contribute to microglia-mediated inflammation in the CNS.

\section{Materials and methods}

\subsection{Lipidomics analysis}

Lipid analysis was performed as previously reported [33]. Prior to lipid extraction, internal standards were added corresponding to each lipid class, lipids were then extracted from whole cells by a modified Bligh and Dyer method, samples were dried under nitrogen and resuspended in chloroform. Immediately prior to injection the extracted lipids were combined with 2:1 methanol:chloroform with $0.1 \%(\mathrm{v} / \mathrm{v})$ ammonium hydroxide. This was injected directly into a Q-TOF 2 mass spectrometer (Waters, Milford, MA) using a nano-esi spray source at $1 \mu \mathrm{l} / \mathrm{min}$. Spectra were obtained in positive-ion mode $\left(\mathrm{PC}+\mathrm{H}^{+}, \mathrm{TAG}+\mathrm{NH}_{4}^{+}\right)$and negative-ion mode $\left(\mathrm{FFA}-\mathrm{H}^{+}, \mathrm{PA}-\mathrm{H}^{+}\right.$, $\left.\mathrm{PE}-\mathrm{H}^{+}, \mathrm{PG}-\mathrm{H}^{+}, \mathrm{PI}-\mathrm{H}^{+}, \mathrm{PS}-\mathrm{H}^{+}, \mathrm{CL}-2 \mathrm{H}^{+}\right)$. Acquired spectra were centroided using the Masslynx software then deconvoluted and deisotoped with excel macros.

\subsection{Cell culture and treatments}

Murine N9 microglia were cultured in Iscove's Modified Dulbecco's Medium (IMDM) (+ L-glutamine, + 25 mM HEPES) (Gibco) supplemented with $5 \%(\mathrm{v} / \mathrm{v})$ fetal bovine serum (FBS) (Gibco) and $1 \%(\mathrm{v} / \mathrm{v})$ penicillin/streptomycin (Gibco). Subconfluent cells were treated with lipopolysaccharides (LPS) (Escherichia coli, strain 055:B5) (Sigma, \#62326) in medium containing 1\% FBS as described in the figure legends. In experiments with oleic acid, control group cells were treated with bovine serum albumin (BSA) alone $(0.1 \% \mathrm{w} / \mathrm{v})$. Pharmacological inhibitors SP600125 (Calbiochem, \#420119), SB203580 (Sigma, S8307) and LY294002 (Cell Signaling, \#9901) were added $30 \mathrm{~min}$ prior to the addition of LPS $(10 \mu \mathrm{g} / \mathrm{ml})$. For every $10 \mu \mathrm{M}$ of SP600125, $0.1 \%$ of DMSO was added to the cultures, as recommended by the supplier.

\section{3. $O A / B S A$ complex preparation}

OA/BSA complex was formed by mixing $30 \mathrm{mg}$ of sodium oleate (Nu-Check Prep, S-1120) with fatty acid-free BSA solution (5\% w/v) (Sigma, A6003) for $5 \mathrm{~h}$ at $37^{\circ} \mathrm{C}$. After the incubation, the $\mathrm{pH}$ of the solution was adjusted to $\mathrm{pH} 7.4$ and the solution was filtered through a $0.22 \mu \mathrm{m}$ filter. Non-esterified OA concentration in this solution was determined with the NEFA C method kit (Wako). The final molar ratio of OA to BSA was approximately 4.4:1. Aliquots of the stock solution were stored at $-20^{\circ} \mathrm{C}$.

\subsection{Staining of lipid droplets}

LDs in cells fixed with $4 \%$ paraformaldehyde were stained with the neutral lipid-staining fluorophores BODIPY 493/503 (4,4-difluoro1,3,5,7,8-pentamethyl-4-bora-3a,4a-diaza-s-indacene) (Invitrogen, D-3922) or HCS LipidTOX (Invitrogen, H34477). Stock solution of BODIPY 493/503 was made by dissolving the powder in DMSO. Protected from light cells were incubated with BODIPY 493/503 (20 $\mu \mathrm{M}$, diluted in PBS) for 10 min or with HCS LipidTOX (1:200 in PBS) for $30 \mathrm{~min}$ at room temperature. Cells were washed at least twice with PBS.

\subsection{Confocal microscopy}

Images were acquired with a Zeiss LSM 510 NLO inverted confocal microscope using a Plan Achromat 63X/1.4 Oil DIC objective. Microglial cells were seeded on confocal chamber slides (Lab-Tek, Nalge Nunc International, Rochester, NY, USA) at a density of $1.5 \times 10^{4}$ cells/well (area of well $=0.8 \mathrm{~cm}^{2}$ ) or on coverslips $\left(\right.$ area $\left.=1.1 \mathrm{~cm}^{2}\right)$ at a density of $2 \times 10^{4}$ cells/coverlsip. Surfaces were coated with rat tail collagen (Sigma, C7661) prior to seeding. Coverslips were mounted on glass microscope slides (Fisher Scientific, 12-550-14) using glycerol-free mounting media Vectashield H-1000 (Vector) and were sealed around the perimeter with clear nail polish. Images of BODIPY 493/503-labeled LDs were acquired using an Argon $488 \mathrm{~nm}$ excitation laser and a 500-550 band pass (BP) filter. HCS LipidTOX-labeled LDs were detected using the HeNe $633 \mathrm{~nm}$ laser and a long pass (LP) 650 filter. Alexa Fluor 594 (red) was detected using HeNe 543 excitation laser and BP 565-615 IR filter. Each Z-stack image consisted of 10 to 20 optical slices taken at $0.3 \mu \mathrm{m}$ intervals and had dimensions of $73.1 \times 73.1 \times 5.7 \mu \mathrm{m}(\mathrm{x}, \mathrm{y}, \mathrm{z})$ when zoomed by a factor of 2 , and $97.5 \times 97.5 \times 5.7 \mu \mathrm{m}(\mathrm{x}, \mathrm{y}, \mathrm{z})$ when 
zoomed by a factor of 1.5 . All images were acquired at a resolution of $1024 \times 1024$ pixels.

\subsection{Analysis of $L D$ volume and number}

Confocal Z-stack images of fluorescently labeled LDs were analyzed using the software Imaris (Bitplane). LDs were visualized as point-like structures by selecting "Spots object". Prior to spot (i.e. LD) detection, the background of the image was subtracted. The threshold of spot detection was adjusted such that only spots associated with lipid droplets were considered in the analysis. The number, radius $(\mu \mathrm{m})$, and volume $\left(\mu \mathrm{m}^{3}\right)$ of LDs were calculated by the software. A minimum of 9 fields were analyzed for each condition. The number of LDs per cell was calculated by dividing the number of LDs in the field by the number of cells present in the field. Data computed in Imaris were used to calculate the size distribution in the total LD population that included all LDs detected by microscopy. Small (diameter $<0.5 \mu \mathrm{m}$ ), medium (diameter $>0.5<1.0 \mu \mathrm{m}$ ) and large (diameter $>1 \mu \mathrm{m}$ ) LDs in their different populations were determined using the histogram analysis in Microsoft Excel's (2007).

\subsection{Nitric oxide (NO) production}

Release of NO from microglia in cultures was measured using the Griess reagent (Sigma, G-4410). Cells seeded at a density of $2 \times 10^{5}$ / well in 24-well culture plates (Sarstedt \#83.1836) were treated with LPS $(10 \mu \mathrm{g} / \mathrm{ml})$ or OA $(50 \mu \mathrm{M})$ for $24 \mathrm{~h}$ in $1 \%$ FBS-containing media. At the end of treatments, supernatants $(50 \mu \mathrm{l})$ were collected and incubated with the Griess reagent $(50 \mu \mathrm{l})$ for $15 \mathrm{~min}$, after which the absorbance at $540 \mathrm{~nm}$ was measured with a spectrophotometer. In each experiment, a standard curve with different concentrations of nitrite $\left(\mathrm{NO}_{2}^{-}\right)$was used to calculate the concentration of $\mathrm{NO}$ detected in the culture media. Each measurement was carried out in quadruplicate samples from two independent experiments.

\subsection{Western blot analysis}

Whole cell extracts made by lysing the cells in NP-40 buffer (50 mM Tris pH 8.0, $137 \mathrm{mM} \mathrm{NaCl}, 1 \% \mathrm{NP}-40,10 \%$ glycerol) were supplemented with complete protease inhibitor cocktail (Roche Applied Science) (1 tablet per $25 \mathrm{ml}$ of lysis buffer) and with phosphatase inhibitors sodium orthovanadate $(1 \mathrm{mM})$ and sodium fluoride $(1 \mathrm{mM})$. Cell lysates were boiled in $6 \times$ sample buffer (12\% SDS, $30 \%$ glycerol, $0.2 \%$ bromophenol blue (w/v), 12\% 2-mercaptoethanol, $0.375 \mathrm{M}$ Tris $\mathrm{HCl} \mathrm{pH} \mathrm{6.8)} \mathrm{at} \mathrm{a} \mathrm{5:1} \mathrm{(cell} \mathrm{lysate:sample} \mathrm{buffer)} \mathrm{ratio} \mathrm{for} 5 \mathrm{~min}$, and proteins were resolved by SDS-PAGE and then transferred $(1 \mathrm{~h}$, $100 \mathrm{~V}$, in ice-cold transfer buffer) to nitrocellulose membranes (Hybond, Amersham Bioscience). Blocking of the membranes was performed by incubating them in $5 \%$ milk or BSA (in TBS-T). Membranes were incubated with primary antibodies to perilipin-2 (guinea pig polyclonal) (1:2000) (Fitzgerald Ind. 20R-AP002), JNK (rabbit polyclonal) (1:1000) (Santa Cruz, sc-571), phospho-JNK (rabbit monoclonal) (1:1000) (Cell Signaling, \#4668), p38 (rabbit polyclonal) (1:500) (Santa Cruz, sc-535), phospho-p38 (rabbit monoclonal) (1:1000) (Cell Signaling \#9215), cPLA $2-\alpha$ (mouse monoclonal (1:500) (Santa Cruz, sc-454, 4-4B-3 C) or actin (mouse monoclonal) (1:1000) (Millipore, MAB1501R) overnight at $4{ }^{\circ} \mathrm{C}$. Following incubation with primary antibodies, membranes were incubated with horse radish peroxidise-linked secondary antibodies anti-rabbit IgG (Amersham Biosciences, NA 934), anti-guinea pig IgG (Sigma, A5545) and antimouse IgG (GE Healthcare, NXA931). The binding of the secondary antibody to the primary antibody was visualized by using an ECL Plus detection kit and HyBlot autoradiography films (Denville). Films were scanned (greyscale at 16 -bits) and relative intensities of the immunoreactive bands were analyzed using the gel analyzer tool in the software Image J (1.42).

\subsection{Immunocytochemistry of perilipin-2 and $C P L A_{2}-\alpha$}

Microglia were seeded at a density of $1.5 \times 10^{4}$ cells/well on coverslips (Fisher) coated with rat tail collagen (Sigma, C7661). Paraformaldehydefixed cells were permeablized with Triton X-100 (0.1\%) (Amersham Biosciences, \# 17-1315-01) for $5 \mathrm{~min}$ and blocked with goat serum (10\%) (Sigma, G9023) for $1 \mathrm{~h}$ for perilipin-2 or $2 \mathrm{~h}$ for $\mathrm{CPLA}_{2}-\alpha$ experiments. Immunostaining was performed by incubating the cells with a primary antibody to perilipin-2 (guinea pig polyclonal, 1:200) (Fitzgerald Ind. 20R-AP002) or $\mathrm{CPLA}_{2}-\alpha$ (mouse monoclonal, 1:100) (Santa Cruz, sc-454, 4-4B-3C). Primary antibodies were targeted with anti-guinea pig (goat) (Invitrogen, A11076) or anti-mouse (goat) (Invitrogen, A11005) Alexa Fluor 594-conjugated secondary antibodies. Coverslips were mounted on glass microscope slides (Fisher Scientific, 12-550-14) using glycerol-free mounting media Vectashield H-1000 (Vector).

\subsection{MTT assay for cell viability}

The MTT assay was performed as described previously [9]. N9 microglia were seeded in 24 -well plates $\left(5 \times 10^{4}\right.$ cells/well, area per well $=2 \mathrm{~cm}^{2}$ ) (Sarstedt, 83.1836), cultured overnight and then treated with the inhibitors, oleic acid and LPS for $12 \mathrm{~h}$ as described in the figure legends. Mitochondrial metabolic activity in viable cells was determined by conversion of MTT (thiazolyl blue tetrazolium bromide) (M2128, Sigma) to formazan. The absorbance from dissolved formazan in DMSO was measured spectrophotometrically at $594 \mathrm{~nm}$.

\subsection{Statistical analysis}

Statistical analysis was performed using Systat (version 13). Comparisons between multiple groups were done by ANOVA, followed by post hoc tests such as Tukey's or Dunnett's. T-tests were used when comparing only two groups. All data are presented as group means \pm SEM. The Fischer exact test (two-tail) was used to test for differences in proportions of small, medium and large LDs between their different populations in $2 \times 2$ tables. In all statistical tests, values of $\mathrm{p}<0.05$ were considered significant.

\section{Results}

\section{1. $L P S$ induces $L D$ accumulation, alters $L D$ size distribution and increases triglyceride content}

Binding of LPS to TLR4 leads to the activation of TLR4 signal transduction pathways known to induce NO synthase expression. Therefore, we measured the concentration of NO in the culture media following LPS $(10 \mu \mathrm{g} / \mathrm{ml})$ treatment for 6 and $24 \mathrm{~h}$. LPS treatment resulted in a dramatic increase in NO release $(55.5 \pm 1.8 \mu \mathrm{M})$ when compared with untreated cells $(0.4 \pm 0.6 \mu \mathrm{M})(\mathrm{p}<0.005)$ (Supplemental Fig. 1). We sought to assess LD dynamics during microglial activation by examining changes in LD numbers and volumes. Using confocal microscopy, we monitored the dynamics of microglial LDs, which were visualized with the neutral lipid-selective fluorescent dye BODIPY 493/503. Cells treated with LPS $(10 \mu \mathrm{g} / \mathrm{ml})$ for $12 \mathrm{~h}$ displayed significantly elevated number of LDs, as compared to nonstimulated microglial cells (Fig. 1A). OA, a monounsaturated long chain fatty acid, is known to be a potent inducer of LDs [7] and was therefore used as a positive control. We observed a marked accumulation of LDs in cells supplemented with OA $(50 \mu \mathrm{M})$ for $12 \mathrm{~h}$ (Fig. 1A). In contrast to LPS, however, OA did not stimulate NO release from microglia (Supplemental Fig. 1). The number of LDs per cell (\#LD/cell) was determined at each of the different timepoints $(3,6$, 9, 12 and $24 \mathrm{~h}$ ) following LPS or OA treatment. LPS caused a timedependent increase in \#LD/cell, with a maximum number observed 
A
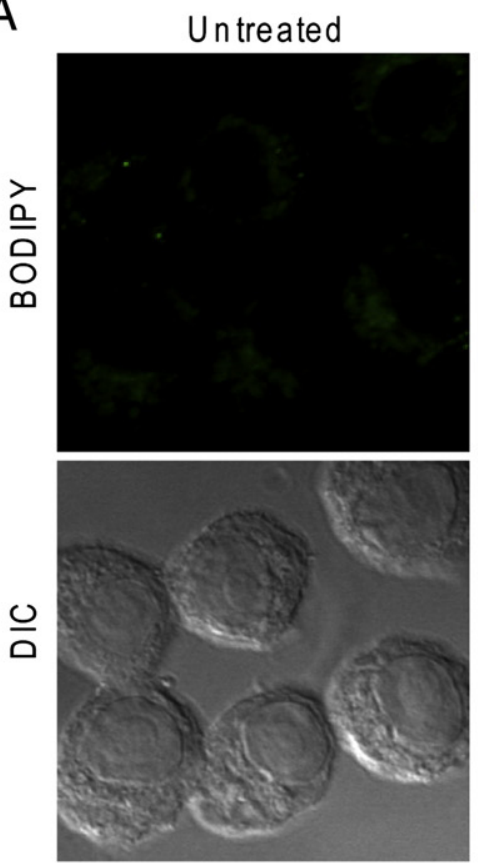

B

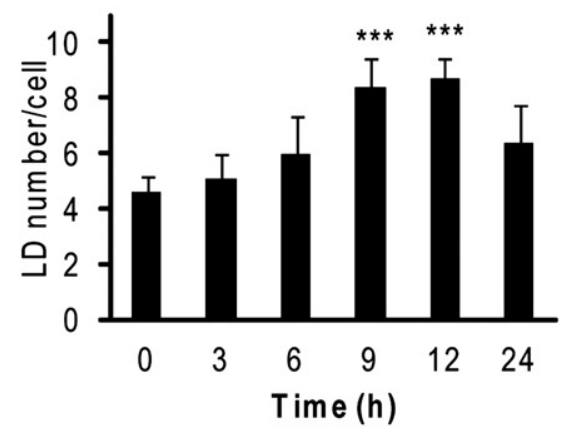

LPS
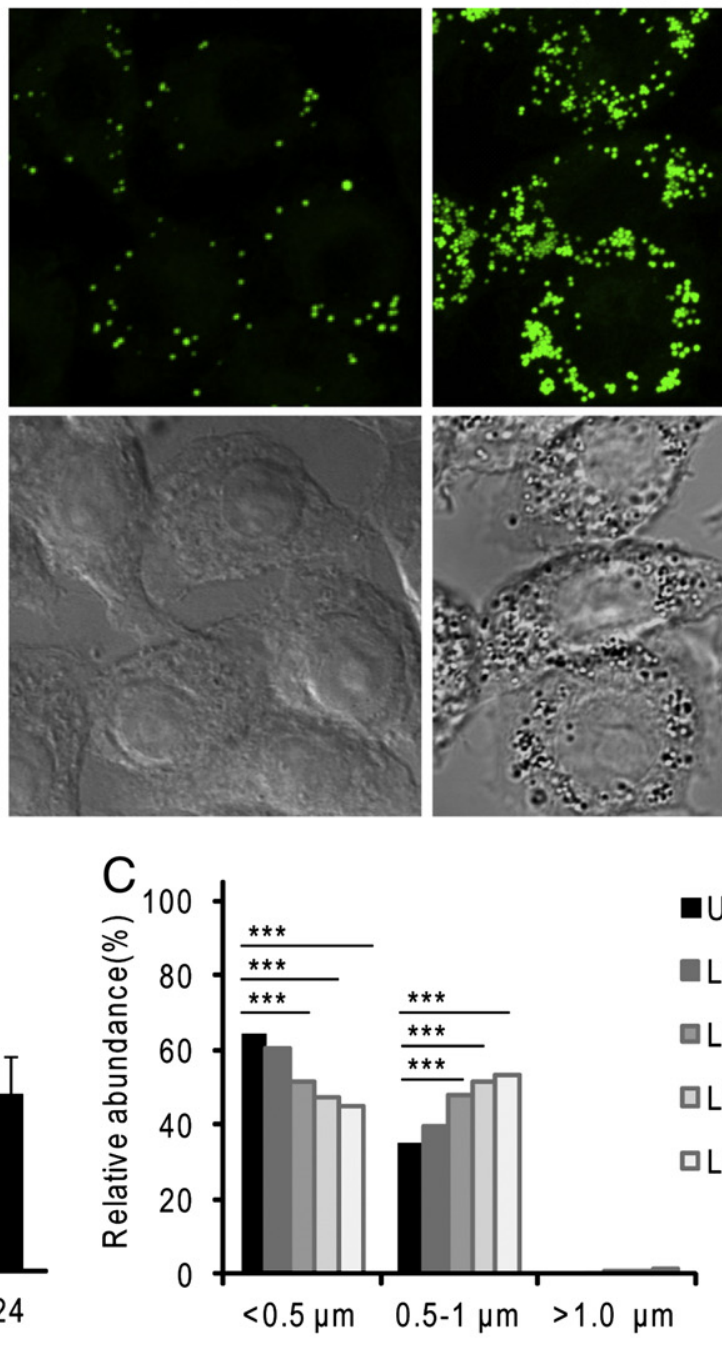

Oleic acid

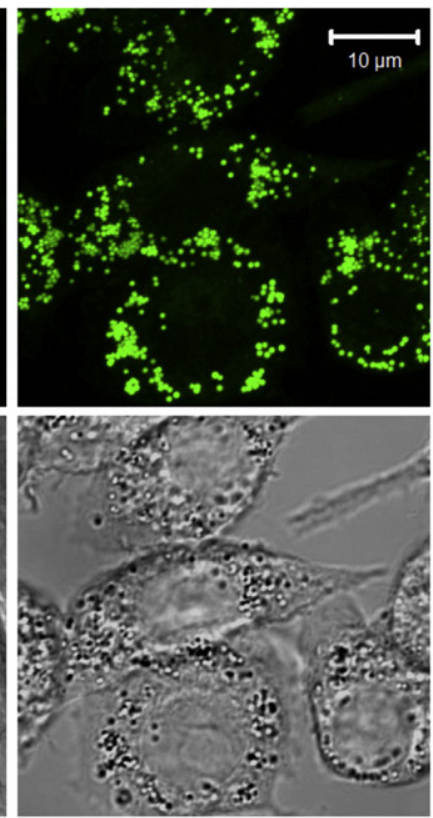

Untreated

LPS $3 h$

LPS $6 h$

口LPS $9 \mathrm{~h}$

DLPS $12 \mathrm{~h}$
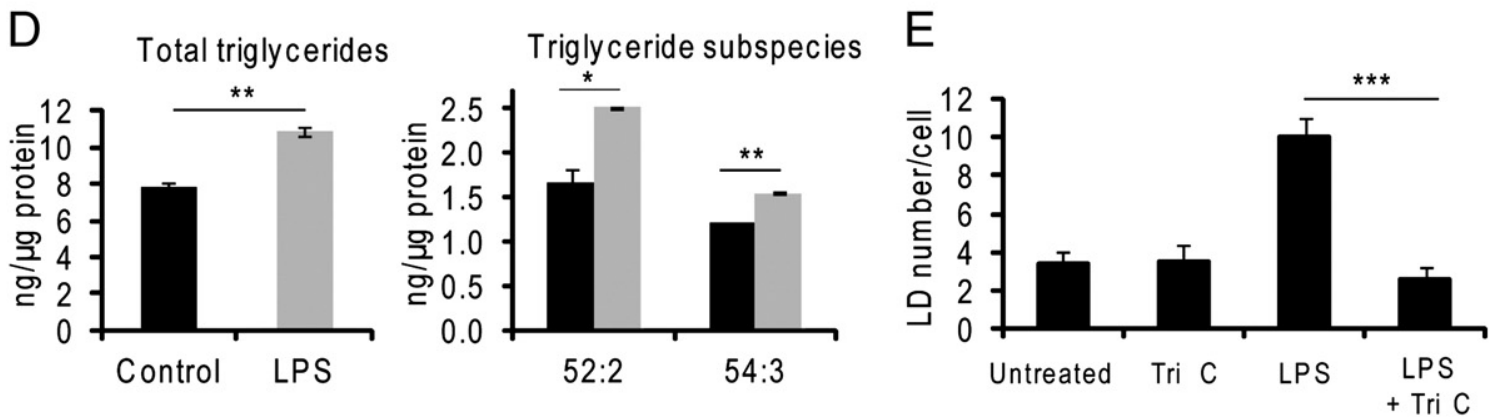

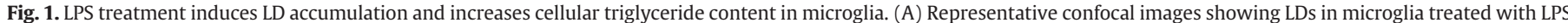

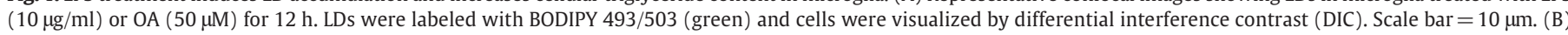

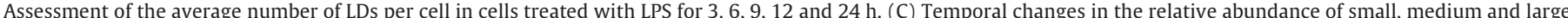

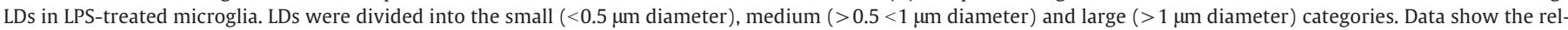

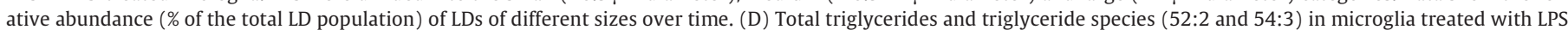

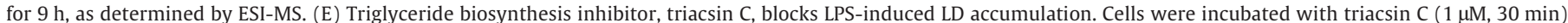
and treated with LPS for $9 \mathrm{~h}$. Statistically significant differences are indicated by ${ }^{*}(\mathrm{p}<0.05),{ }^{* *}(\mathrm{p}<0.01)$ and ${ }^{* * *}(\mathrm{p}<0.005)$

by $12 \mathrm{~h}$ of LPS treatment ( $8.7 \pm 0.7$ vs. $4.6 \pm 0.6$ in untreated cells) $(\mathrm{p}<0.05)$ (Fig. 1B). OA treatment rapidly increased LD content in microglial cells, causing a $~ 16$-fold increase in \#LD/cell $(113 \pm 13$ in treated vs. $7 \pm 2$ in untreated) after $3 \mathrm{~h}(\mathrm{p}<0.005)$ (Supplemental Fig. 2A). Typically, LDs are heterogeneous with respect to their sizes, as well as their lipid [34,35] and protein content [36]. In N9 microglia, LDs with a diameter ranging from $\sim 250 \mathrm{~nm}$ to $2 \mu \mathrm{m}$ or even greater can be detected by confocal microscopy (our unpublished observations). In addition to an induction of LD number by inflammatory stimuli, an increase in LD size has also been seen in inflammatory 
macrophages [37]. Therefore, we assessed the effect of LPS on the size distribution of LDs in microglial cells. After $12 \mathrm{~h}$ of LPS treatment, we observed a significant reduction $(-19.8 \%, \mathrm{p}<0.005)$ in the relative abundance of small LDs, accompanied by an increase in the relative abundance of medium $(+18.6 \%, \mathrm{p}<0.005)$ and large $(+1.2 \%$, not significant) LDs (Fig. 1C). OA exerted a pronounced effect on LD size distributions (Supplemental Fig. 2B). A reduced relative abundance of small LDs $(-26 \%, \mathrm{p}<0.005)$ and concomitant increase of medium and large LDs ( $24 \%, \mathrm{p}<0.005$ and $2 \%, \mathrm{p}<0.005$, respectively) were seen within three hours (Supplemental Fig. 2B). These findings imply that LPS alters LD size distribution in microglia, although to a much lesser extent than OA. Our data show that LPS-activated microglia have a higher LD content than untreated microglial cells and that LPS and OA greatly differ in the extent to which they induce LD accumulation and alter LD sizes.

To further demonstrate that LPS leads to LD accumulation, we performed mass spectrometry-based lipidomic analysis to determine changes in the levels of triglycerides (TG), the major neutral lipids found in LDs. Following their extraction from microglia treated with LPS for 9 h, TG levels were measured by quantitative electrospray ionization mass spectrometry (ESI-MS). Cells treated with LPS displayed a significant increase in total cellular TG levels (Fig. 1D) and in TG species 52:2 and 54:3, thereby supporting the observed increase in LD content in activated microglia. TG synthesis requires incorporation of longchain fatty acyl CoAs into glycerol-3-phosphate, monoacylglycerol and diacylglycerol [38]. Triacsin C, an inhibitor of long-chain acyl-CoA synthetase (ACSL), inhibits de novo synthesis of TG from glycerol and diacylglycerol [39]. Treatment with triacsin C abolished the increase in LDs induced by LPS, suggesting that de novo synthesis of TGs was required under this condition (Fig. 1E and Supplemental Fig. 1B).
3.2. LPS-induces activation of JNK and p38 MAPK and stimulates perilipin-2 expression

JNK and p38 MAPKs are key players in LPS-induced inflammatory response of microglia [40]. JNK activation leads to transcriptional activation of AP-1, a heterodimeric transcriptional complex [26]. It has been reported that LPS-induced perilipin-2 expression is in part mediated by an increased binding activity of AP- 1 to the Ets/AP-1 element on the perilipin-2 gene promoter [27]. We first explored the effect of LPS on p38 and JNK by determining the extent of their phosphorylation by Western blot analysis. LPS induced a significant increase in p38 phosphorylation after 15 min of treatment $(p<0.01)$ (Fig. 2A \& B). LPS also caused a rapid (15 min), transient and robust increase in JNK phosphorylation ( $\mathrm{p}<0.005)$ (Fig. 2C \& D). In contrast, neither p38 nor JNK were activated following OA treatment at any timepoint between 0 and 120 min (Supplemental Fig. 2C).

Perilipin-2 has been reported to stimulate neutral lipid accumulation and LD formation in macrophages and fibroblasts [19,41]. In macrophages, LPS increases perilipin-2 expression leading to LD accumulation $[27,28]$. To date, the role of perilipin-2 has not been investigated in neural cells. We hypothesized that activation with LPS will increase perilipin-2 protein levels in microglia. Following LPS stimulation, perilipin-2 levels increased in a time-dependent manner with a peak at $9 \mathrm{~h}$ after LPS addition corresponding to a $\sim 3$-fold increase $(\mathrm{p}<0.05)$ over the control (Fig. 3A \& B). Immunostaining experiments of perilipin-2 indicated that the protein colocalizes with LDs in LPStreated cells (Fig. 3C). In the experiments with our positive control, we observed a significant increase $(>3$-fold, $\mathrm{p}<0.05$ ) in perilipin-2 expression after $3 \mathrm{~h}$ of $\mathrm{OA}$ treatment (Supplemental Fig. $3 \mathrm{~A} \& \mathrm{~B}$ ).

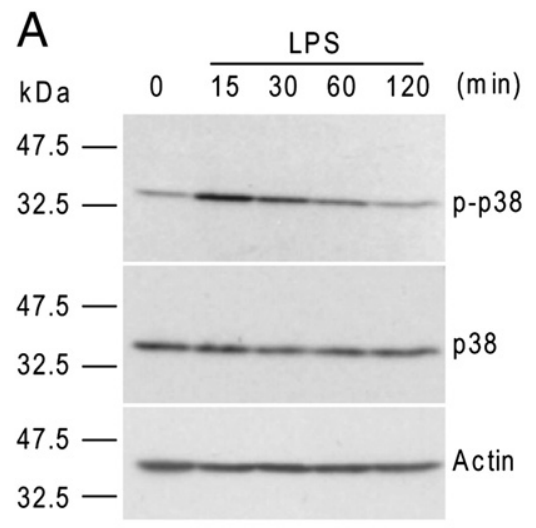

B
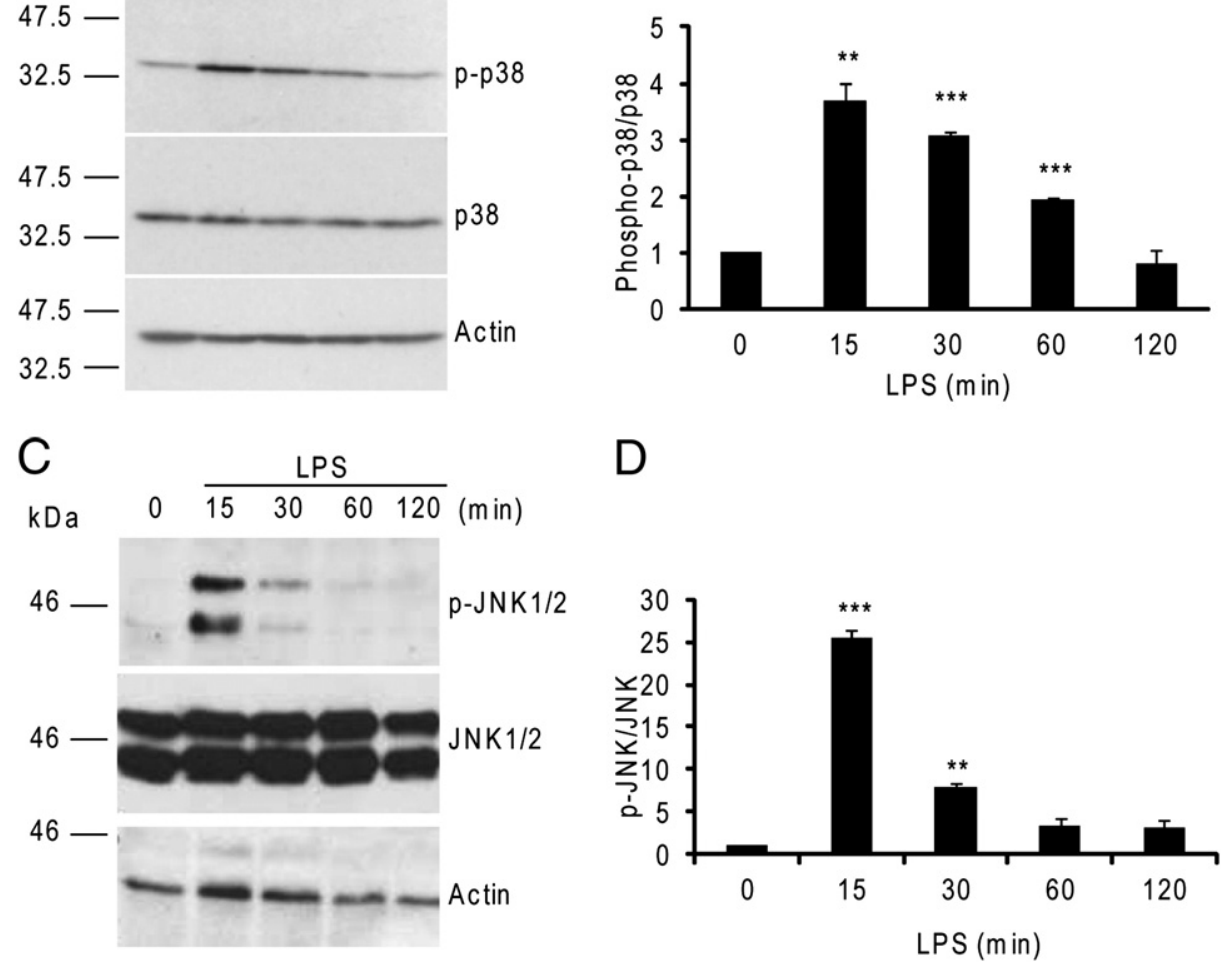

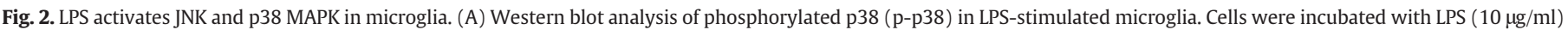

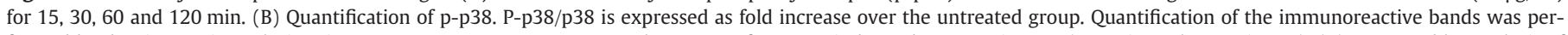

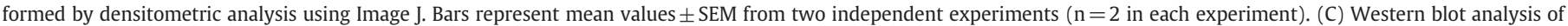

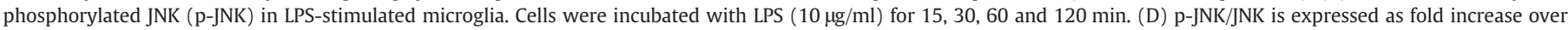

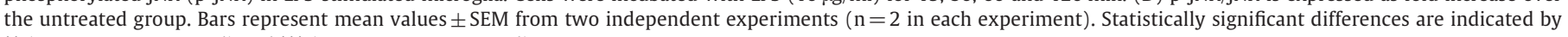
** $(\mathrm{p}<0.01$, vs. untreated $)$ and ${ }^{* * *}(\mathrm{p}<0.005$, vs. untreated $)$. 
Moreover, perilipin-2 extensively colocalized with LDs (Supplemental Fig. 3C). Altogether, the above data imply that LPS rapidly activates MAPK pathways and leads to an increase in perilipin-2 expression in microglia.

3.3. LPS-induced LD formation is blocked by inhibitors of $p 38$ and PI3K, but not by an inhibitor of JNK

To investigate whether MAPK activation regulates LD formation, LPS-stimulated microglia were treated with selective inhibitors of JNK (SP600125) and p38 (SB203580). We found that in the presence of LPS, SP600125 $(10 \mu \mathrm{M})$ further increased the LD number/cell (Fig. 4A
\& B). In contrast, SB203580 $(10 \mu \mathrm{M})$ reduced the LD number/cell $(\mathrm{p}<0.005)$ (Fig. 4A \& B). However, SB203580 also reduced the LD number/cell under basal conditions $(p<0.005)$. We also examined whether inhibition of p38 downregulates perilipin-2 expression in LPS-stimulated microglia. Indeed, SB203580 treatment, though not SP600125 treatment, significantly decreased perilipin-2 expression in LPS-treated microglia (Fig. 4C). It has been demonstrated that SB203580, when used at concentrations above $2-3 \mu \mathrm{M}$, can block the phosphorylation and activation of Akt/protein kinase B (PKB) by inhibiting 3-phosphoinositide-dependent protein kinase 1 (PDK1) [42]. Akt/PKB, a serine-threonine kinase is activated by growth factors and plays a central role in cell survival, growth and proliferation. Interestingly, Akt/PKB was also shown to promote
A

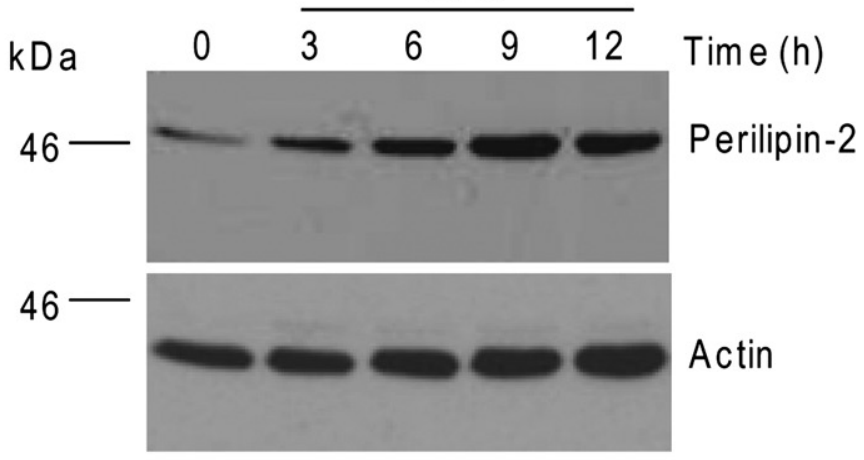

B
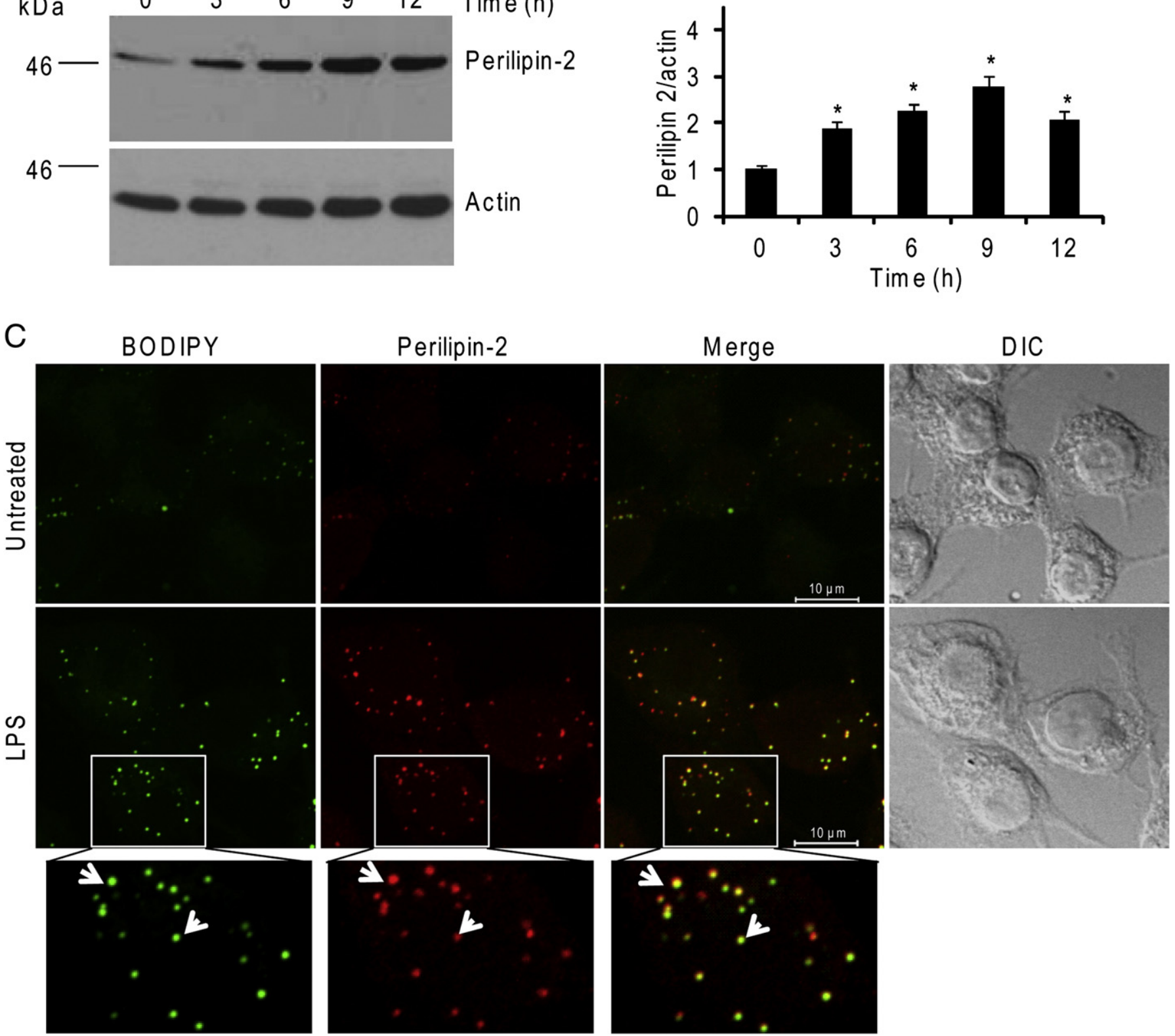

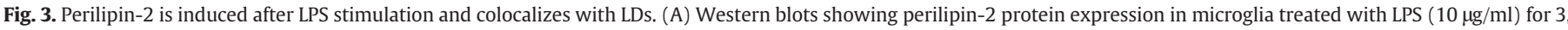

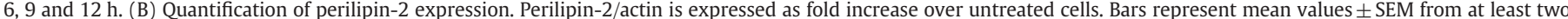

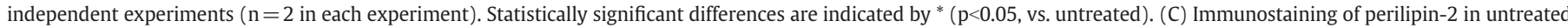

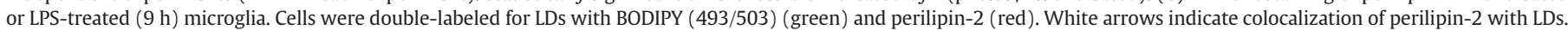
Scale bar $=10 \mu \mathrm{m}$. 
A
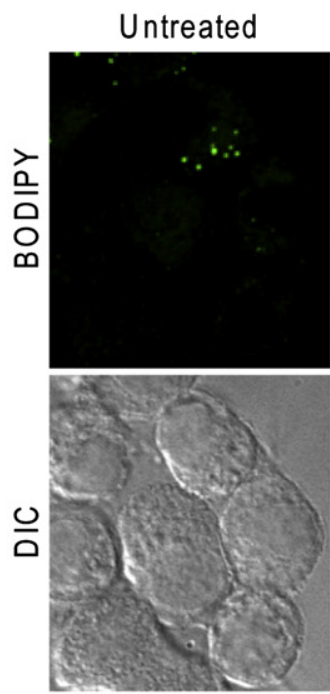

LPS
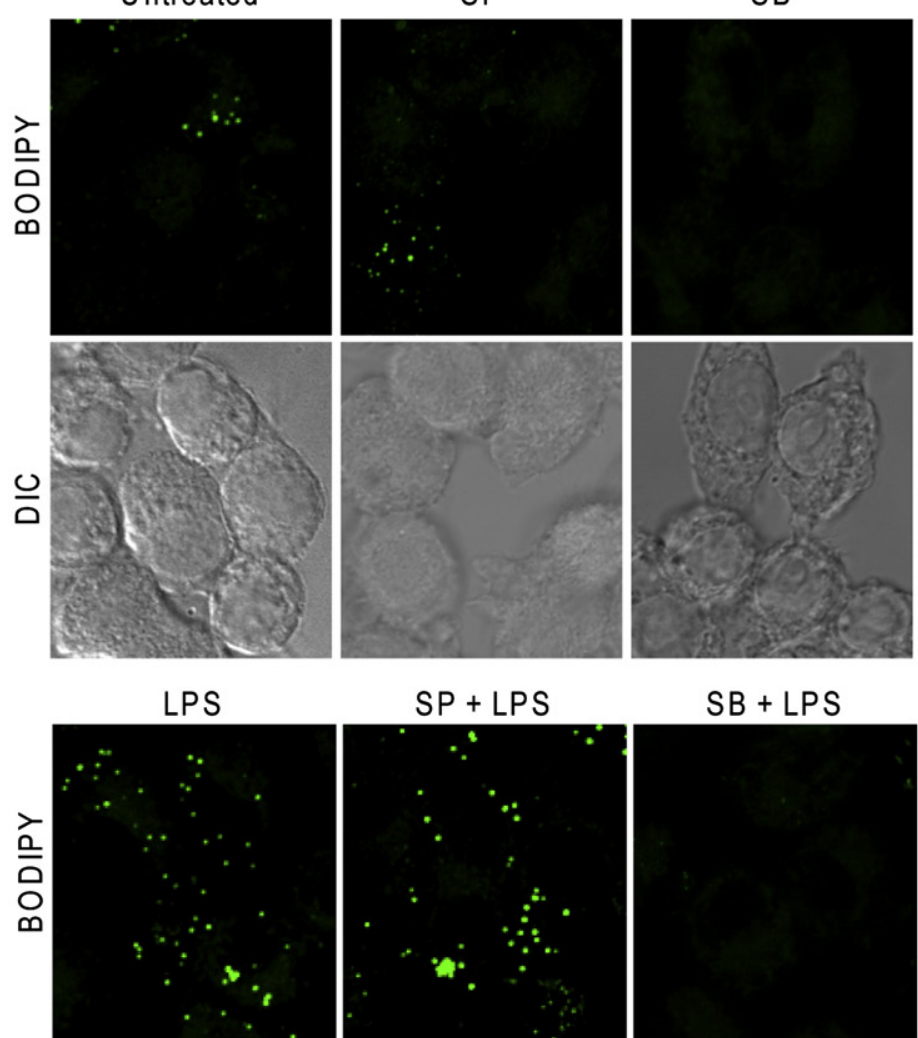

SP + LPS
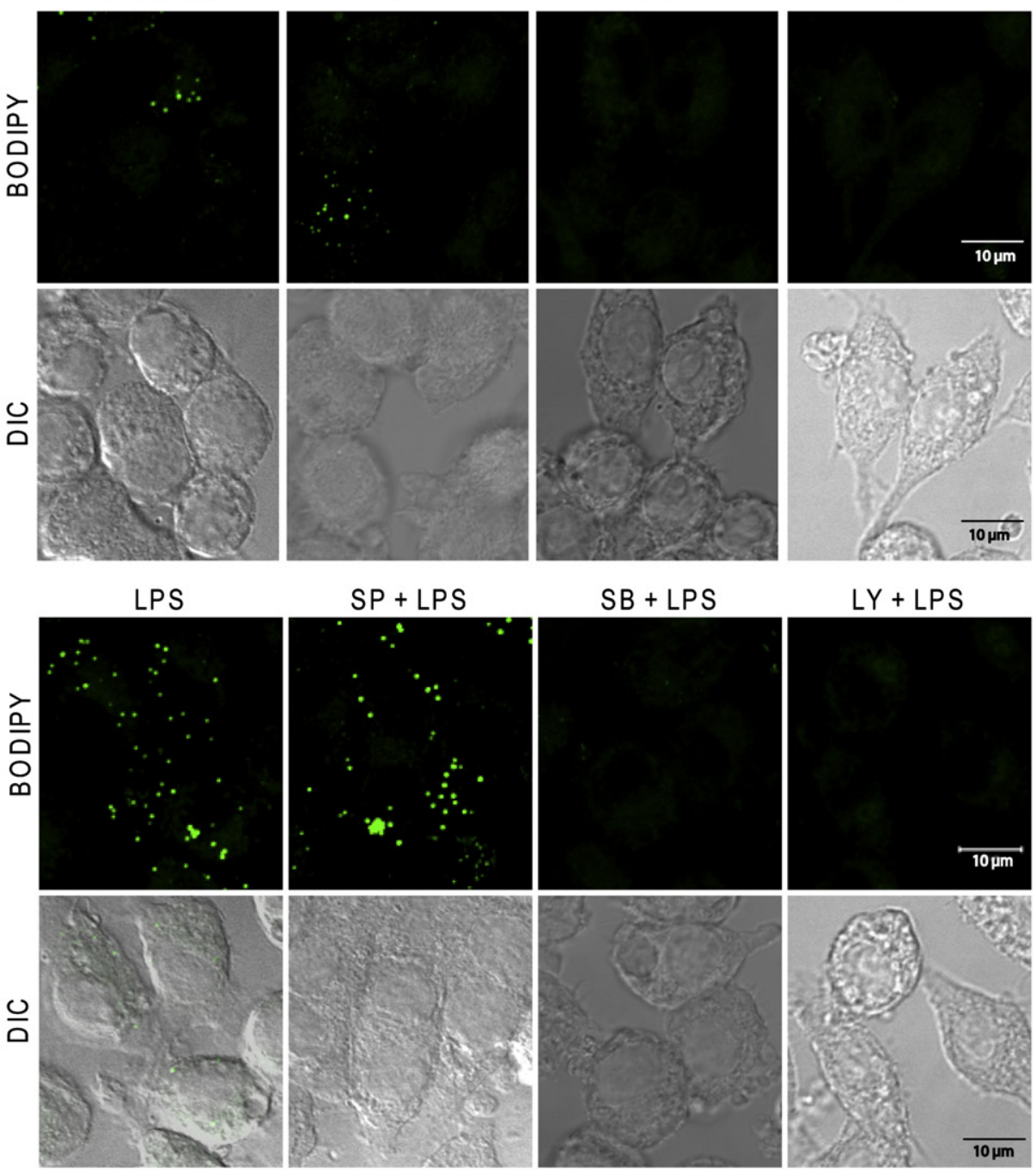

B

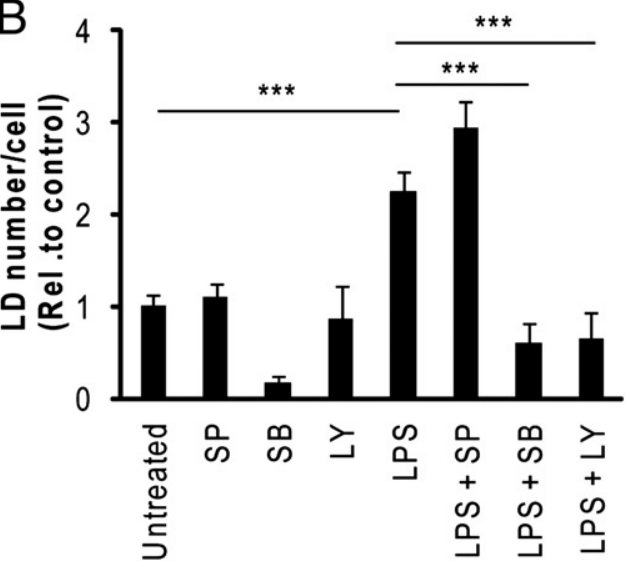

C

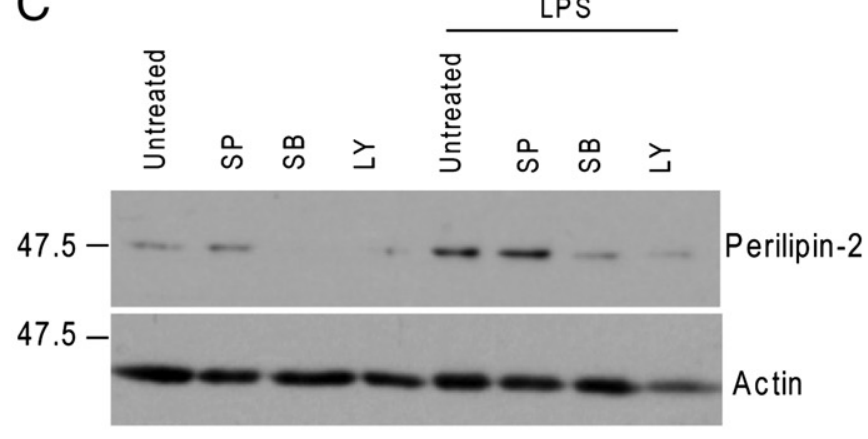

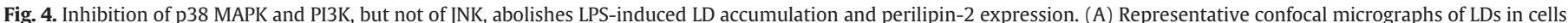

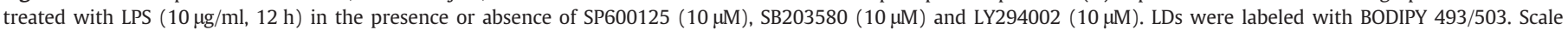

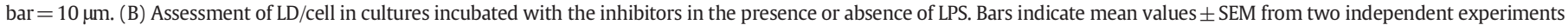

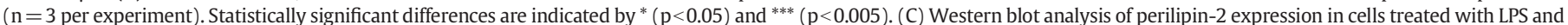

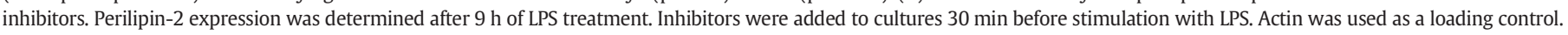

accumulation of LDs in Drosophila nurse cells [43]. To examine whether Akt/PKB is a regulator of LD formation, we treated microglia with an inhibitor (LY294002) of class I phosphatidylinositol 3-kinase (PI3K), an upstream activator of Akt/PKB. LPS-induced LD accumulation in microglia was blunted by LY294002 treatment ( $<$ <0.005) (Fig. 4A \& B). The inhibitor also reduced perilipin-2 expression (Fig. 4C). Together, these data implicate the p38 MAPK and PI3K/Akt pathways in the regulation of LD formation in LPS-treated microglial cells. 
3.4. $p 38$ and PI3K inhibitors do not block OA-induced LD formation, but decrease the size of $L D s$

Roles for p38 MAPK and PI3K activation in OA-induced LD formation have been previously reported in epithelial cells [15]. To examine whether p38 and PI3K regulate OA-induced LDs in microglia, we tested their effect on LD formation by confocal microscopy. SB203580 or LY294002 did not block the induction of LDs by OA (Supplemental Fig. $5 \mathrm{~A} \& \mathrm{~B}$ ), but they did markedly decrease the average size (volume) of LDs ( $\mathrm{p}<0.01$ ) (Supplemental Fig. 5C).

3.5. In microglia, cytosolic phospholipase $A_{2}\left(C P L A_{2}\right)$ colocalizes with LPS-induced LDs, but not with OA-induced LDs

LDs in leukocytes were previously shown to contain significant amounts of esterified arachidonate in their triglyceride and phospholipid pools [13]. Different eicosanoid-synthesizing enzymes, including the key enzyme for the release of arachidonic acid, $\mathrm{CPLA}_{2}-\alpha$, compartmentalize at LDs in human monocytic (U937) and rat intestinal (IEC-6) epithelial cells $[13,15]$. We asked whether or not the newly formed LDs in LPS-treated microglia play a function in eicosanoid synthesis. To address this question, we examined by confocal microscopy the colocalization of $\mathrm{CPLA}_{2}-\alpha$ with LDs in LPS-treated microglia. Colocalization of $C P L A_{2}-\alpha$ with LDs could not be assessed in untreated cells, because LDs were almost entirely absent (Fig. 5A). LPS induced the formation of many LDs which colocalized with $\mathrm{CPLA}_{2}-\alpha$ (Fig. $5 \mathrm{~A}$, bottom row). In contrast, OA-induced LDs did not colocalize with $\mathrm{CPLA}_{2}-\alpha$. Treatment with LPS or OA did not alter the protein levels of $\mathrm{CPLA}_{2}-\alpha$ (Fig. $5 \mathrm{~B}$ ).

\section{Discussion}

In this report we show that LPS-induced TLR4 activation in microglia is accompanied by marked changes in intracellular lipid droplet (LD) status as manifested by an increase in the number and volume of LDs in the cells. LPS also increased the expression of perilipin-2, a major LD surface-associated protein. We attribute this effect of LPS on LDs to the activation of p38 MAPK, since inhibition of p38 suppressed LPSinduced perilipin-2 expression and LD formation. Taken together, our results suggest that LDs are actively involved in LPS-induced inflammatory response in microglia.

We first demonstrated that LPS-mediated TLR4 activation in microglia leads to LD accumulation. In vivo studies in mice have demonstrated that LD number in leukocytes is increased following LPS administration [12]. Because of their implication in the innate immune response, LDs were described as "inflammatory organelles" that can serve as key markers of cell activation [44] We showed that in LPSchallenged microglia, the increase in the LD number was significant. However, temporal analysis of LDs over a $24 \mathrm{~h}$ period following LPS stimulation demonstrated that the initial increase in LD number does not persist; after reaching a peak following $12 \mathrm{~h}$ of LPS treatment, the number of LDs decreased. This decrease could be explained by increased fusion between LDs, leading to larger, but fewer LDs in the cells. LD fusion is an inducible process [45,46]. In fact, LPS did cause marked changes in the LD size distribution pattern in microglia, shifting the balance towards larger LDs and reducing the abundance of small-sized LDs. We speculate that the enlargement of LDs in LPS-stimulated microglia was caused, at least in part, by activated homotypic fusion of their smaller forms. Treatment with LPS resulted in a significant increase in cellular TG levels in microglia. The effect of LPS on lipid synthesis in macrophages has been previously examined $[47,48]$. It was demonstrated that macrophages (RAW 264.7) treated with LPS accumulate TG, but not cholesterol esters (CE) [48]. An in vivo study showed that LPS increased both TG and CE levels in peritoneal macrophages [47]. In microglia, LD accumulation triggered by LPS required TG biosynthesis, since triacsin C, an inhibitor of long-chain acyl-CoA synthetase (ACSL) activity, blocked the induction of LDs by LPS. Whether CE levels increase concurrently with TG levels in LPS-treated microglia should also be determined in future studies.

In parallel with LPS, we supplemented microglia with OA, a dietary monounsaturated fatty acid. As expected, OA strongly induced LD formation and caused marked changes in the pattern of LD size distribution. Upon entering the cell, unsaturated free fatty acids (FFAs) are rapidly activated by becoming converted to their CoA esters. TGs consist of three fatty acid chains attached to a glycerol backbone by ester bonds. In the final reaction of TG synthesis, a fatty acyl-CoA and diacylglycerol are covalently attached in an esterification reaction catalyzed by the enzyme acyl-CoA:diacylglycerol acyltransferase (DGAT) [49]. The esterification-driven conversion of FFAs into stored TGs is considered a protective mechanism against FFA-induced lipotoxicity [50]. In the present study, OA treatment in microglia did not induce cytotoxicity and displayed no activating effect on JNK and p38 MAPK.

We further demonstrated that LPS treatment increases perilipin-2 protein expression. The increase in perilipin-2 expression took place before the increase in the number of LDs, suggesting that new perilipin-2 protein synthesis was required for LPS-induced LD formation. In fact, using actinomycin D and cycloheximide, Gu et al. demonstrated that in RAW 264.7 macrophages LPS-induced perilipin-2 expression was stimulated at the transcriptional level and was mediated by new protein synthesis [27]. Post-translational mechanisms were shown to regulate perilipin-2 via the ubiquitin/proteasome proteolytic pathway [21]. Increased cellular neutral lipids (e.g triglyceride) stabilize perilipin-2 and prevent its degradation by the proteasome [51]. Therefore, similar mechanisms could contribute to increased perilipin-2 levels in microglia accumulating TGs.

We observed a robust induction of perilipin-2 in OA-supplemented microglia. Fatty acids can bind peroxisome proliferator activated receptors (PPAR) $\alpha$ - and $-\gamma$ and thereby regulate gene expression [52]. A PPAR response element (PPRE) is located in the promoter region of perilipin-2 [53] and regulates perilipin-2 expression in OA-treated cells [54]. OA was also shown to affect the post-translational regulation of perilipin-2 (ADRP). Indeed, OA treatment reduced levels of polyubiquitinated perilipin-2 and prevented its proteolytic degradation [21].

JNK and p38 MAPKs play an important role in the pro-inflammatory actions of microglia and macrophages $[55,56]$. Their activity is highly regulated by TLR4-generated signals. TLR4 agonists, such as LPS, induce homodimerization of the TLR4/MD-2 complex and trigger a downstream signaling cascade involving IRAK and TAK1. This leads to activation of MAPK kinases (MKK) 3, 4, 6 and 7 [26]. MKK3/6 mainly phosphorylate $\mathrm{p} 38$, whereas MKK 4/7 phosphorylate JNK. Phosphorylation of c-Jun and ATF2 by JNK and p38 increase the transcriptional activity of AP-1, leading to expression of inflammatory cytokines [26]. The effects of LPS on microglia have been previously investigated $[57,58]$. In BV2 microglia, LPS-induced activation involves iNOS expression, NFKB activation, p38 phosphorylation, reactive oxygen species production and cyclooxygenase -2 expression [57]. Interestingly, OA showed an anti-inflammatory effect by reducing the proinflammatory effects of LPS. In agreement with these studies, we showed that in N9 microglia LPS caused rapid and transient stimulation of JNK and p38 MAPK and increased NO production. OA treatment had virtually no stimulating effect on JNK, p38 and NO release. Taken together, these findings reveal differential effects of $\mathrm{OA}$ and LPS on the TLR4-initiated signal transduction pathway. 


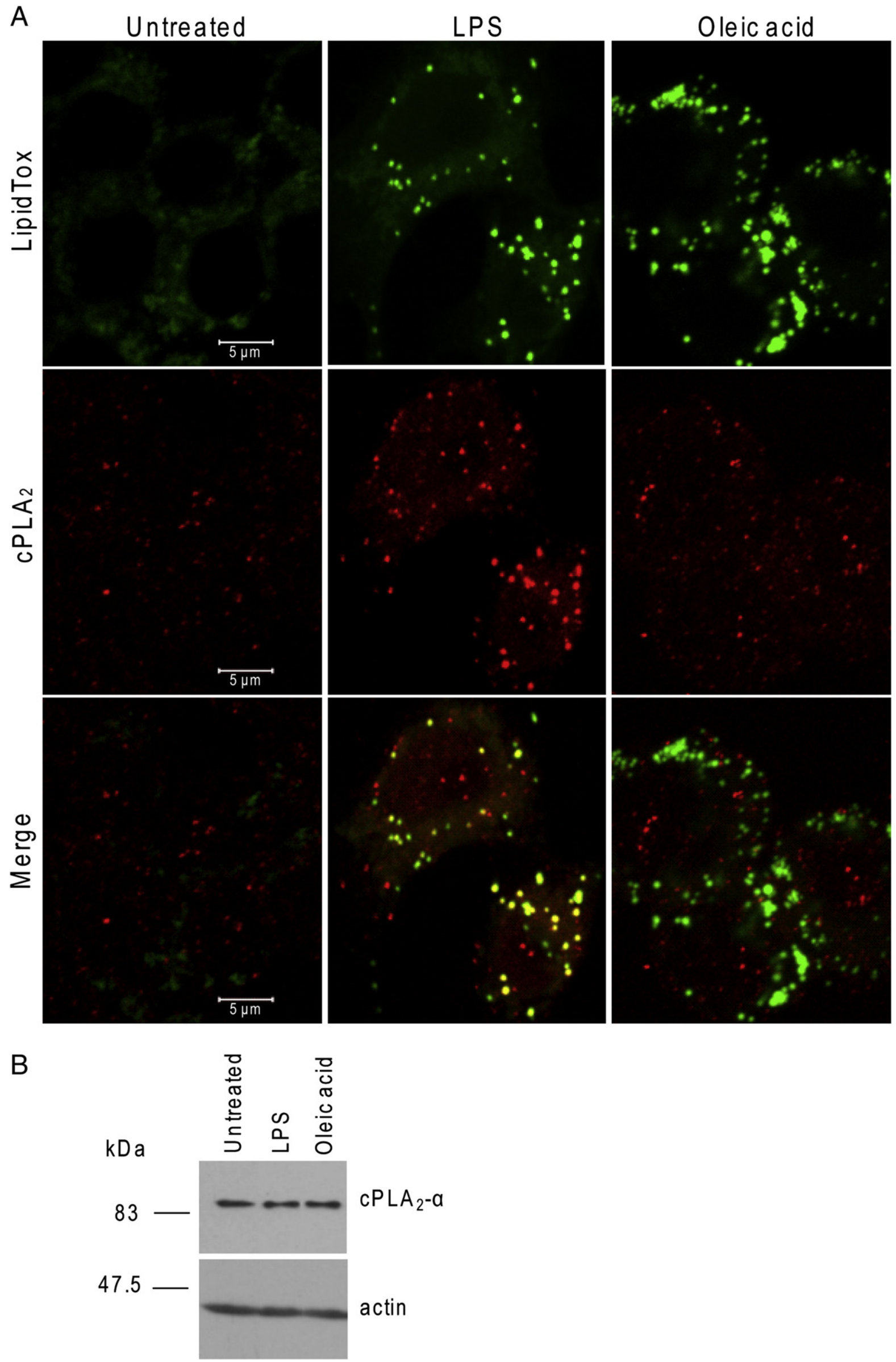




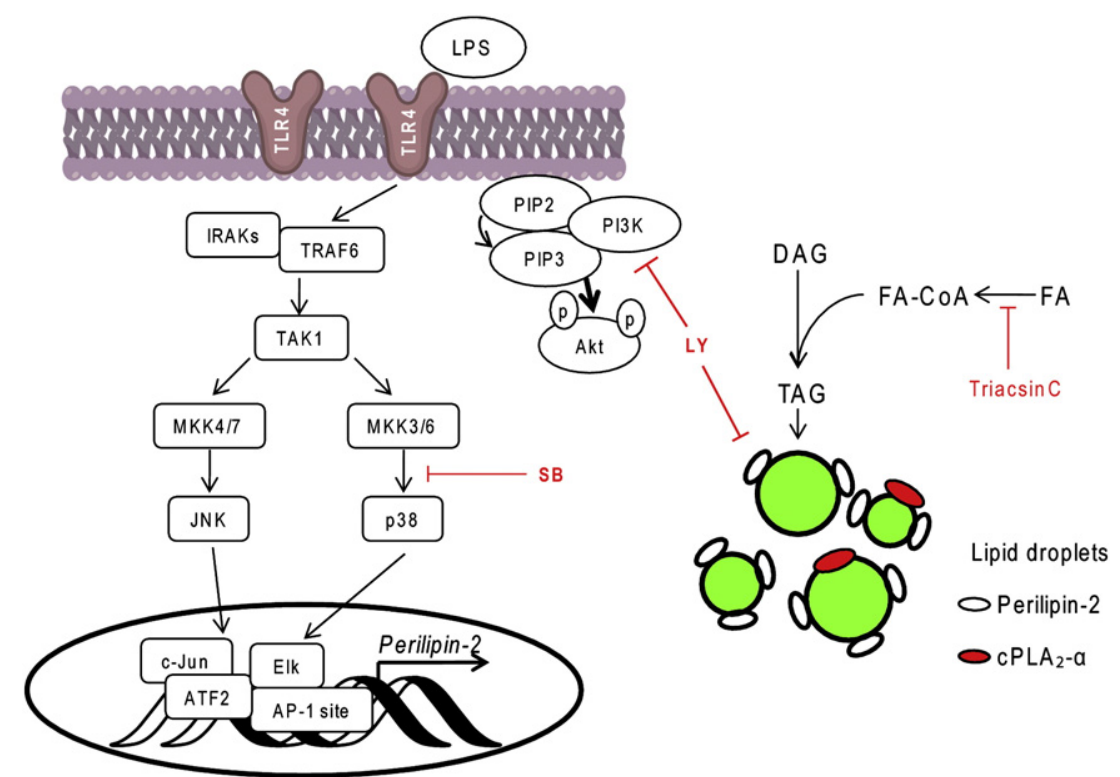

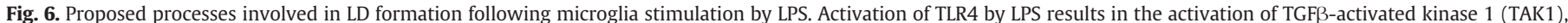

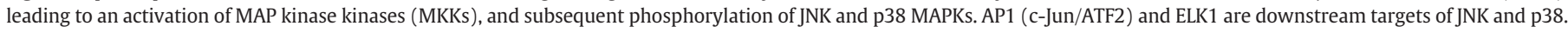

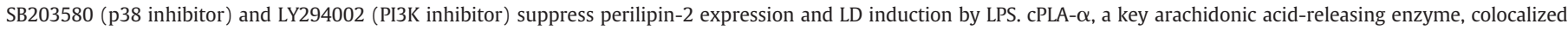
with LDs. The induction of LDs by LPS was blocked by triacsin C, an inhibitor of triglyceride biosynthesis.

We demonstrated that the inhibition of p38 MAPK or PI3K, but not the inhibition of JNK, downregulates LPS-induced perilipin-2 expression and LD accumulation. SP600125 inhibits JNK 1/2 with high specificity [59]. SP600125 treatment further increased LD formation in LPSstimulated microglia, contrary to our expectations. The growing body of evidence implies the existence of a crosstalk between JNK and other kinases. Activation of the PI3K/Akt pathway and ERK1/2 following inhibition of JNK with SP600125 have been reported in macrophages and neurons $[40,60]$. Of note, ERK $1 / 2$ and Akt/PI3K signaling pathways were implicated in regulating LD formation in macrophages [61]. It is conceivable, therefore, that under LPS treatment, pharmacological inhibition of JNK causes a compensatory activation of either ERK1/2 or Akt/PI3K pathway, leading to increased LD formation. The inhibitor of 38 MAPK, SB203580, abolished the increase in perilipin-2 expression and LD accumulation caused by LPS treatment. It was previously shown that SB203580 inhibits LPS-induced activation of p38 MAPK and transcription factor AP-1 in macrophages [62]. Considering that LPS can enhance perilipin- 2 promoter activity by activating AP-1, the suppression of peilipin-2 by SB203580 in LPS-treated microglia could have been mediated by the inhibition of AP-1. In the absence of LPS, SB203580 also reduced the number of $L D$. This could have been caused by the offtarget inhibitory effect of SB203580 on the Akt/PKB signaling pathway, as previously reported in experiments applying the inhibitor at concentrations above $2-3 \mu \mathrm{M}$ [42]. In sum, our study revealed that both LPS and OA can stimulate LD formation and perilipin-2 expression in microglia, but with different dynamics and to a remarkably different extent. $\mathrm{OA}$ is a much more potent inducer of LD formation and perilipin-2 expression, as compared to LPS. Our results reveal at least two distinct signaling pathways involved in LD formation in microglia. Whereas OA induces LD formation in a MAPK independent manner, LPS induces LD accumulation by strongly activating p38 (Fig. 6). The presence of colocalization between $\mathrm{CPLA}_{2}-\alpha$ and LPS-induced LDs indicates a potential role for these organelles in the regulation of eicosanoid synthesis in activated microglia.

We suggest that LDs induced by LPS play an important regulatory role in the microglial inflammatory response. Altogether, our findings imply that in the central nervous system, specific changes in the biogenesis of LDs and their proteins may contribute to regulation of pathologies associated with inflammation.
Supplementary materials related to this article can be found online at doi: 10.1016/j.bbalip.2012.01.007.

\section{Acknowledgements}

We acknowledge the financial assistance from Canadian Institute of Health Research (CIHR) (DM). VIT is a Concordia University Research Chair in Genomics, Cell Biology and Aging. Centre for Biological Applications of Mass Spectrometry at Concordia University is acknowledged for outstanding services. VRR is supported by a scholarship from the CIHR. This work was also supported by the Concordia University Chair Fund (VIT). We thank Dr. Garabet Yeretssian for his careful and critical reading.

\section{References}

[1] D.J. Murphy, The biogenesis and functions of lipid bodies in animals, plants and microorganisms, Prog. Lipid Res. 40 (2001) 325-438.

[2] K. Tauchi-Sato, S. Ozeki, T. Houjou, R. Taguchi, T. Fujimoto, The surface of lipid droplets is a phospholipid monolayer with a unique fatty acid composition, J. Biol. Chem. 277 (2002) 44507-44512.

[3] M. Bell, H. Wang, H. Chen, J.C. McLenithan, D.W. Gong, R.Z. Yang, D. Yu, S.K. Fried M.J. Quon, C. Londos, C. Sztalryd, Consequences of lipid droplet coat protein downregulation in liver cells: abnormal lipid droplet metabolism and induction of insulin resistance, Diabetes 57 (2008) 2037-2045.

[4] W. Motomura, M. Inoue, T. Ohtake, N. Takahashi, M. Nagamine, S. Tanno, Y. Kohgo, T. Okumura, Up-regulation of ADRP in fatty liver in human and liver steatosis in mice fed with high fat diet, Biochem. Biophys. Res. Commun. 340 (2006) 1111-1118.

[5] B.H. Chang, L. Chan, Regulation of triglyceride metabolism. III. Emerging role of lipid droplet protein ADFP in health and disease, Am. J. Physiol. 292 (2007) G1465-G1468.

[6] P.T. Bozza, J.P. Viola, Lipid droplets in inflammation and cancer, Prostaglandins. Leukot. Essent. Fatty Acids 82 (2010) 243-250.

[7] Y. Fujimoto, J. Onoduka, K.J. Homma, S. Yamaguchi, M. Mori, Y. Higashi, M. Makita, T. Kinoshita, J. Noda, H. Itabe, T. Takanoa, Long-chain fatty acids induce lipid droplet formation in a cultured human hepatocyte in a manner dependent of Acyl-CoA synthetase, Biol. Pharm. Bull. 29 (2006) 2174-2180.

[8] A.R. Silva, P. Pacheco, A. Vieira-de-Abreu, C.M. Maya-Monteiro, B. D'Alegria, K.G. Magalhaes, E.F. de Assis, C. Bandeira-Melo, H.C. Castro-Faria-Neto, P.T. Bozza, Lipid bodies in oxidized LDL-induced foam cells are leukotriene-synthesizing organelles: a MCP-1/CCL2 regulated phenomenon, Biochim. Biophys. Acta 1791 (2009) 1066-1075.

[9] A. Khatchadourian, D. Maysinger, Lipid droplets: their role in nanoparticleinduced oxidative stress, Mol. Pharm. 6 (2009) 1125-1137.

[10] A. Gubern, M. Barcelo-Torns, J. Casas, D. Barneda, R. Masgrau, F. Picatoste, J. Balsinde, M.A. Balboa, E. Claro, Lipid droplet biogenesis induced by stress involves triacylglycerol 
synthesis that depends on group VIA phospholipase A2, J. Biol. Chem. 284 (2009) 5697-5708.

[11] P.T. Bozza, K.G. Magalhaes, P.F. Weller, Leukocyte lipid bodies - biogenesis and functions in inflammation, Biochim. Biophys. Acta 1791 (2009) 540-551.

[12] P. Pacheco, F.A. Bozza, R.N. Gomes, M. Bozza, P.F. Weller, H.C. Castro-Faria-Neto, P.T. Bozza, Lipopolysaccharide-induced leukocyte lipid body formation in vivo: innate immunity elicited intracellular Loci involved in eicosanoid metabolism, J. Immunol. 169 (2002) 6498-6506.

[13] W. Yu, P.T. Bozza, D.M. Tzizik, J.P. Gray, J. Cassara, A.M. Dvorak, P.F. Weller, Cocompartmentalization of MAP kinases and cytosolic phospholipase A2 at cytoplasmic arachidonate-rich lipid bodies, Am. J. Pathol. 152 (1998) 759-769.

[14] P.F. Weller, A.M. Dvorak, Lipid bodies: intracellular sites for eicosanoid formation, J. Allergy Clin. Immunol. 94 (1994) 1151-1156.

[15] L.S. Moreira, B. Piva, L.B. Gentile, F.P. Mesquita-Santos, H. D'Avila, C.M. MayaMonteiro, P.T. Bozza, C. Bandeira-Melo, B.L. Diaz, Cytosolic phospholipase A2-driven PGE2 synthesis within unsaturated fatty acids-induced lipid bodies of epithelial cells, Biochim. Biophys. Acta 1791 (2009) 156-165.

[16] H.Y. Qi, J.H. Shelhamer, Toll-like receptor 4 signaling regulates cytosolic phospholipase A2 activation and lipid generation in lipopolysaccharide-stimulated macrophages, J. Biol. Chem. 280 (2005) 38969-38975.

[17] P.E. Bickel, J.T. Tansey, M.A. Welte, PAT proteins, an ancient family of lipid droplet proteins that regulate cellular lipid stores, Biochim. Biophys. Acta 1791 (2009) 419-440.

[18] A.R. Kimmel, D.L. Brasaemle, M. McAndrews-Hill, C. Sztalryd, C. Londos, Adoption of PERILIPIN as a unifying nomenclature for the mammalian PAT-family of intracellular lipid storage droplet proteins, J. Lipid Res. 51 (2010) 468-471.

[19] G. Larigauderie, C. Furman, M. Jaye, C. Lasselin, C. Copin, J.C. Fruchart, G. Castro, M. Rouis, Adipophilin enhances lipid accumulation and prevents lipid efflux from THP-1 macrophages: potential role in atherogenesis, Arterioscler. Thromb. Vasc. Biol. 24 (2004) 504-510.

[20] H. Robenek, S. Lorkowski, M. Schnoor, D. Troyer, Spatial integration of TIP47 and adipophilin in macrophage lipid bodies, J. Biol. Chem. 280 (2005) 5789-5794.

21] G. Xu, C. Sztalryd, X. Lu, J.T. Tansey, J. Gan, H. Dorward, A.R. Kimmel, C. Londos, Post-translational regulation of adipose differentiation-related protein by the ubiquitin/proteasome pathway, J. Biol. Chem. 280 (2005) 42841-42847.

[22] U.K. Hanisch, H. Kettenmann, Microglia: active sensor and versatile effector cells in the normal and pathologic brain, Nat. Neurosci. 10 (2007) 1387-1394.

[23] W.Y. Chan, S. Kohsaka, P. Rezaie, The origin and cell lineage of microglia: new concepts, Brain Res. Rev. 53 (2007) 344-354.

[24] M.L. Block, L. Zecca, J.S. Hong, Microglia-mediated neurotoxicity: uncovering the molecular mechanisms, Nat. Rev. Neurosci. 8 (2007) 57-69.

[25] M.M. Buchanan, M. Hutchinson, L.R. Watkins, H. Yin, Toll-like receptor 4 in CNS pathologies, J. Neurochem. 114 (2010) 13-27.

[26] E.F. Wagner, A.R. Nebreda, Signal integration by JNK and p38 MAPK pathways in cancer development, Nat. Rev. 9 (2009) 537-549.

[27] J.Q. Gu, S. Ikuyama, P. Wei, B. Fan, J. Oyama, T. Inoguchi, J. Nishimura, Pycnogenol an extract from French maritime pine, suppresses Toll-like receptor 4-mediated expression of adipose differentiation-related protein in macrophages, Am. J. Physiol. Endocrinol. Metab. 295 (2008) E1390-E1400.

[28] K.R. Feingold, M.R. Kazemi, A.L. Magra, C.M. McDonald, L.G. Chui, J.K. Shigenaga, S.M. Patzek, Z.W. Chan, C. Londos, C. Grunfeld, ADRP/ADFP and Mal1 expression are increased in macrophages treated with TLR agonists, Atherosclerosis 209 (2010) 81-88

[29] C.S. McKimmie, D. Roy, T. Forster, J.K. Fazakerley, Innate immune response gene expression profiles of N9 microglia are pathogen-type specific, J. Neuroimmunol. 175 (2006) 128-141.

[30] O. Quehenberger, A.M. Armando, A.H. Brown, S.B. Milne, D.S. Myers, A.H. Merrill S. Bandyopadhyay, K.N. Jones, S. Kelly, R.L. Shaner, C.M. Sullards, E. Wang, R.C. Murphy, R.M. Barkley, T.J. Leiker, C.R. Raetz, Z. Guan, G.M. Laird, D.A. Six, D.W. Russell, J.G. McDonald, S. Subramaniam, E. Fahy, E.A. Dennis, Lipidomics reveals a remarkable diversity of lipids in human plasma, Journal of lipid research 51 (2010) 3299-3305.

[31] P. Wei, S. Taniguchi, Y. Sakai, M. Imamura, T. Inoguchi, H. Nawata, S. Oda, Y. Nakabeppu, J. Nishimura, S. Ikuyama, Expression of adipose differentiation-related protein (ADRP) is conjointly regulated by PU.1 and AP-1 in macrophages, J. Biochem. 138 (2005) 399-412.

[32] J.M. Medina, A. Tabernero, Astrocyte-synthesized oleic acid behaves as a neurotrophic factor for neurons, J. Physiol. Paris 96 (2002) 265-271.

[33] S.D. Bourque, V.I. Titorenko, A quantitative assessment of the yeast lipidome using electrospray ionization mass spectrometry, J. Vis. Exp. 30 (2009), doi:10.3791/1513.

[34] H.A. Rinia, K.N. Burger, M. Bonn, M. Muller, Quantitative label-free imaging of lipid composition and packing of individual cellular lipid droplets using multiplex CARS microscopy, Biophys. J. 95 (2008) 4908-4914.

[35] R. Bartz, W.H. Li, B. Venables, J.K. Zehmer, M.R. Roth, R. Welti, R.G. Anderson, P. Liu, K.D. Chapman, Lipidomics reveals that adiposomes store ether lipids and mediate phospholipid traffic, Journal of lipid research 48 (2007) 837-847.

[36] B.K. Straub, P. Stoeffel, H. Heid, R. Zimbelmann, P. Schirmacher, Differential pattern of lipid droplet-associated proteins and de novo perilipin expression in hepatocyte steatogenesis, Hepatology 47 (2008) 1936-1946 Baltimore, MD.

[37] R.C. Melo, D.L. Fabrino, F.F. Dias, G.G. Parreira, Lipid bodies: structural markers of inflammatory macrophages in innate immunity, Inflamm. Res. 55 (2006) 342-348

[38] K.K. Buhman, H.C. Chen, R.V. Farese Jr., The enzymes of neutral lipid synthesis, J. Biol. Chem. 276 (2001) 40369-40372.

[39] R.A. Igal, P. Wang, R.A. Coleman, Triacsin C blocks de novo synthesis of glycerolipids and cholesterol esters but not recycling of fatty acid into phospholipid: evidence for functionally separate pools of acyl-CoA, Biochem. J. 324 (Pt 2) (1997) 529-534.
[40] E. Sanchez-Tillo, M. Comalada, J. Xaus, C. Farrera, A.F. Valledor, C. Caelles, J. Lloberas, A. Celada, JNK1 Is required for the induction of Mkp1 expression in macrophages during proliferation and lipopolysaccharide-dependent activation, J. Biol. Chem. 282 (2007) 12566-12573.

[41] M. Imamura, T. Inoguchi, S. Ikuyama, S. Taniguchi, K. Kobayashi, N. Nakashima, H. Nawata, ADRP stimulates lipid accumulation and lipid droplet formation in murine fibroblasts, Am. J. Physiol. Endocrinol. Metab. 283 (2002) E775-E783.

[42] F.V. Lali, A.E. Hunt, S.J. Turner, B.M. Foxwell, The pyridinyl imidazole inhibitor SB203580 blocks phosphoinositide-dependent protein kinase activity, protein kinase $\mathrm{B}$ phosphorylation, and retinoblastoma hyperphosphorylation in interleukin2-stimulated T cells independently of p38 mitogen-activated protein kinase, J. Biol. Chem. 275 (2000) 7395-7402.

[43] N. Vereshchagina, C. Wilson, Cytoplasmic activated protein kinase Akt regulates lipid-droplet accumulation in Drosophila nurse cells, Development 133 (2006) 4731-4735 (Cambridge, England).

[44] H. D'Avila, C.M. Maya-Monteiro, P.T. Bozza, Lipid bodies in innate immune response to bacterial and parasite infections, Int. Immunopharmacol. 8 (2008) 1308-1315.

[45] P. Bostrom, L. Andersson, M. Rutberg, J. Perman, U. Lidberg, B.R. Johansson, J. FernandezRodriguez, J. Ericson, T. Nilsson, J. Boren, S.O. Olofsson, SNARE proteins mediate fusion between cytosolic lipid droplets and are implicated in insulin sensitivity, Nat. Cell Biol. 9 (2007) 1286-1293.

[46] S. Murphy, S. Martin, R.G. Parton, Quantitative analysis of lipid droplet fusion: inefficient steady state fusion but rapid stimulation by chemical fusogens, PLoS One 5 (2010) e15030.

[47] E.N. Posokhova, O.M. Khoshchenko, M.I. Chasovskikh, E.N. Pivovarova, M.I. Dushkin, Lipid synthesis in macrophages during inflammation in vivo: effect of agonists of peroxisome proliferator activated receptors alpha and gamma and of retinoid $\mathrm{X}$ receptors, Biochemistry 73 (2008) 296-304.

[48] J.L. Funk, K.R. Feingold, A.H. Moser, C. Grunfeld, Lipopolysaccharide stimulation of RAW 264.7 macrophages induces lipid accumulation and foam cell formation, Atherosclerosis 98 (1993) 67-82.

[49] C.L. Yen, S.J. Stone, S. Koliwad, C. Harris, R.V. Farese Jr., Thematic review series: glycerolipids. DGAT enzymes and triacylglycerol biosynthesis, Journal of lipid research 49 (2008) 2283-2301.

[50] L.L. Listenberger, X. Han, S.E. Lewis, S. Cases, R.V. Farese Jr., D.S. Ory, J.E. Schaffer, Triglyceride accumulation protects against fatty acid-induced lipotoxicity, Proc. Natl. Acad. Sci. U. S. A. 100 (2003) 3077-3082.

[51] Y. Masuda, H. Itabe, M. Odaki, K. Hama, Y. Fujimoto, M. Mori, N. Sasabe, J. Aoki, H. Arai, T. Takano, ADRP/adipophilin is degraded through the proteasome-dependent pathway during regression of lipid-storing cells, Journal of lipid research 47 (2006) 87-98

[52] S.A. Kliewer, S.S. Sundseth, S.A. Jones, P. Brown, G.B. Wisely, C.S. Koble, P. Devchand, W. Wahli, T.M. Willson, J.M. Lenhard, J.M. Lehmann, Fatty acids and eicosanoids regulate gene expression through direct interactions with peroxisome proliferatoractivated receptors alpha and gamma, Proc. Natl. Acad. Sci. U. S. A. 94 (1997) 4318-4323.

[53] P. Targett-Adams, M.J. McElwee, E. Ehrenborg, M.C. Gustafsson, C.N. Palmer, J. McLauchlan, A PPAR response element regulates transcription of the gene for human adipose differentiation-related protein, Biochim. Biophys. Acta 1728 (2005) 95-104.

[54] B. Fan, S. Ikuyama, J.Q. Gu, P. Wei, J. Oyama, T. Inoguchi, J. Nishimura, Oleic acidinduced ADRP expression requires both AP-1 and PPAR response elements, and is reduced by Pycnogenol through mRNA degradation in NMuLi liver cells, Am. J. Physiol. Endocrinol. Metab. 297 (2009) E112-E123.

[55] V. Waetzig, K. Czeloth, U. Hidding, K. Mielke, M. Kanzow, S. Brecht, M. Goetz, R. Lucius, T. Herdegen, U.K. Hanisch, c-Jun N-terminal kinases (JNKs) mediate proinflammatory actions of microglia, Glia 50 (2005) 235-246.

[56] J.C. Lee, J.T. Laydon, P.C. McDonnell, T.F. Gallagher, S. Kumar, D. Green, D. McNulty, M.J. Blumenthal, J.R. Heys, S.W. Landvatter, et al., A protein kinase involved in the regulation of inflammatory cytokine biosynthesis, Nature 372 (1994) 739-746.

[57] Y.T. Oh, J.Y. Lee, J. Lee, H. Kim, K.S. Yoon, W. Choe, I. Kang, Oleic acid reduces lipopolysaccharide-induced expression of iNOS and COX-2 in BV2 murine microglial cells: possible involvement of reactive oxygen species, p38 MAPK, and IKK/NF-kappaB signaling pathways, Neurosci. Lett. 464 (2009) 93-97.

[58] H. Zhou, B.M. Lapointe, S.R. Clark, L. Zbytnuik, P. Kubes, A requirement for microglial TLR4 in leukocyte recruitment into brain in response to lipopolysaccharide, J. Immunol. 177 (2006) 8103-8110.

[59] B.L. Bennett, D.T. Sasaki, B.W. Murray, E.C. O'Leary, S.T. Sakata, W. Xu, J.C. Leisten, A. Motiwala, S. Pierce, Y. Satoh, S.S. Bhagwat, A.M. Manning, D.W. Anderson, SP600125, an anthrapyrazolone inhibitor of Jun N-terminal kinase, Proc. Natl. Acad. Sci. U. S. A. 98 (2001) 13681-13686.

[60] M. Yeste-Velasco, J. Folch, G. Casadesus, M.A. Smith, M. Pallas, A. Camins, Neuroprotection by c-Jun NH2-terminal kinase inhibitor SP600125 against potassium deprivation-induced apoptosis involves the Akt pathway and inhibition of cell cycle reentry, Neuroscience 159 (2009) 1135-1147.

[61] P. Pacheco, A. Vieira-de-Abreu, R.N. Gomes, G. Barbosa-Lima, L.B. Wermelinger, C.M. Maya-Monteiro, A.R. Silva, M.T. Bozza, H.C. Castro-Faria-Neto, C. BandeiraMelo, P.T. Bozza, Monocyte chemoattractant protein-1/CC chemokine ligand 2 controls microtubule-driven biogenesis and leukotriene B4-synthesizing function of macrophage lipid bodies elicited by innate immune response, J. Immunol. 179 (2007) 8500-8508.

[62] C. Chen, Y.H. Chen, W.W. Lin, Involvement of p38 mitogen-activated protein kinase in lipopolysaccharide-induced iNOS and COX-2 expression in J774 macrophages, Immunology 97 (1999) 124-129. 